\title{
Ueber Zellen und Nerven der compacten Knochensubstanz.
}

\author{
Von
}

\author{
Hermann Joseph,
}

practischer Arzt in Berlin.

Hierzu Taf. XII.

Bei gewissen Regenerationsversuchen, welche ich am Kopfe von 'Tritonen unternahm, fiel mir der verhältnissmässig grosse Nervenreichthum auf, welcher sich innerhalb der die Schädeldecke bildenden Knochen vorfand. Ich sah die feinen Stämmchen, oft drei bis vier in einem Präparat, die grösseren Blutgefässe begleiten und beschloss ihrem weitern Verlauf nachzuspüren, indessen ich meinen Thieren zunächst allein die Regenerationsarbeit überliess.

$\mathrm{Zu}$ meinen bisherigen Zwecken hatte ich mich der Chromsäure bedient; ich liess sie jetzt im Stich und versuchte die mir schon von Natur genügend präparirt erscheinenden Knochenplättchen, welche das Tritonenhirn umschliessen, zu vergolden. Ich sage umschliessen, weil sowohl die Deckplättchen, wie die Knochen der Schädelbasis zum Vergolden geeignet sind. Die Knochen blieben $1-1 \frac{1}{2}$ Stunden in einer einprocentigen Chlorgoldlösung und kamen dann behuf Reduction in mit einigen Tropfen Essigsäure versetztes Wasser. Anhaftendes Gewebe wurde mit einem feinen Yesserchen uuter der Goldlösung abgeschabt. Nach 24-36 Stunden wurden feine Schnitte mit dem Rasirmesser angefertigt und in Glycerin untersucht.

Die besten Präparate waren die, in denen sich der Inhalt der 
Knochenhöhlen und Havers'schen Kanäle tief violett gefärbt zeigte, während die Grundsubstanz fast völlig farblos, d. h. grauweisslich verblieb. Oft jedoch nahm sie ebenfalls eine mehr oder weniger intensiv bläulich rothe Färbung an und dann blieben unter Umständen die Inhaltsmassen der Höhlen ganz ungefärbt; selten boten sie auch dann ein glänzendes gelbliches homogenes Ansehen dar.

Alle gut gefürbten Präparate wiesen zweifellos ein zelliges Gebilde in der Knochenhöhle nach, das ich zunächst besprechen will.

\section{Die Zellen.}

Die Zelle in der Knochenhöhle bei Tritonen besteht aus dem Zellprotoplasma und einem, selten zweien grossen Zellkernen von rund ovaler Gestalt, mit zahlreichen ziemlich groben, glänzenden und dunkeln Körnchen gefüllt. Curiosität halber erwähne ich, dass ich einmal drei Kerne in einer Zelle sah.

Unter den Körnern des Kerns zeichnen sich die hellglänzenden runden Kernkörperchen aus, meist zwei an der Zahl. Sie sind von ei n em hellen Hof umgeben, wenn sie dicht beisammen liegen, sonst besitzt jedes Kernkörperchen seinen eigenen.

Bald ist das Protoplasma dunkler violett gefärbt, wie der Kern, bald umgekehrt, bald ist die Färbung beider gleich, ohne dass dadurch die scharfe Begrenzung des Kerns verloren geht.

Die immer noch schwankende Ansicht über die Beschaffenheit des Höhleninhalts im völlig ausgebildeten Knochen und namentlich die Ergebnisse der Untersuchungen von Klebs ${ }^{1}$, durch welche dieser zum Verleugnen der Zelle kam, liessen die Anwendung der Goldmethode auf das Knochengewebe verschiedener Thiere gerechtfertigt erscheinen. Ich wählte mir die Schenkelknochen des Frosches, alte Hühner, Meerschweinchen, des Ochsen etc. aus und unterwarf sie derselben Behandlung wie die Schädelknochen der Tritonen, nur dass ich natürlich von grossen Knochen nur Stückchen der kompakten Substanz benutzte.

Von der Knochenschicht, auf welche die Goldlösung und später las angesäuerte Wasser eingewirkt hatte, kann man wiederum feine Schnitte anfertigen.

1) Klebs, Centralblatt für med. Wissenschaft Nัr. 6 1868. Vorl. Mitth. 
In allen bald nach dem Tode des Thieres vergoldeten Präparaten konnte ich Zellen in den Knochenhöhlen nachweisen, welche diese theils ganz erfüllten, theils sich hier und da von der Wandung zurückgezogen hatten. Die Kerne sind rundlich beim Huhn und Meerschweinchen, länglich und schmal beim Ochsen und Frosch; stets sind sie mit einem, häufiger zwei glänzenden Kernkörperchen versehen.

Da, wo sich die Zelle von der Höhlenwand zurückgezogen hat, tinden sich häufig kreisrunde Bläschen, wahrscheinlich Luft. Ich glaube jedoch nicht, dass diese schon ursprünglich vorhanden ist, sondern meine, dass es Kohlensäurebläschen sind, die sich durch die Einwirkung der Essigsäure bilden und in den von der Zelle verlassenen Raum, etwa mit der Flüssigkeit eindringen.

Ob überhaupt zellenfreie Knochenhöhlen in grösserer Ausbreitung vorkommen, ist mir sehr zweifelhaft geworden; denn erstens sieht man in gut und unmittelbar nach dem Tode des Thiers vergoldeten Präparaten in jedem Theile des compacten Ḱnochens die violetten Zellen; zweitens gelingt es längere Zeit nach dem Tode des Thieres noch Zellen dirzustellen oder wo diese schon zerfallen sind, zeigt doch der Inhalt der Knochenhöhle jene violette Beschaffenheit, wie es gewöhnlich dem Protoplisma nach gelungener Vergoldung zukommt, so dass man mindestens einen Zellenrest annehmen muss; drittens ist es mir vorgekommen, wie oben erwähnt, dass der Höhleninhalt ein ganz homogenes Ansehen darbot, ohne jede Spur einer Zelle. Dies beobachte ich namentlich, als ich einmal die Reduction in verdünnter Chromsäure vor sich gehen lassen wollte. Nachträglieh setzte ich einige Tropfen Essigsäure hinzu und bald zeigten sich in den scheinbar mit Flüssigkeit gefüllten Höhlen die schönsten violetten Zellen.

Die Literatur über die Virchow-Donders'schen linochenzellen findet sich so genügend in der Arbeit von E. Neumann ${ }^{1}$ ) angegeben, dass ich nicht nöthig habe, die Freunde und Feinde der Zelle nochmals Alle anzuführen. Nur gegen die Resultate von Klebs ${ }^{2}$ ) die er etwa also formulirt:

1) E. Neumann, Beitrag zur Kenntniss des norm. Zahnbein- und Knochengewebes. Kbg. 1863.

2) Klebs a. a. 0 . 
"Die sternförmigen Höhlen und feinen Ausläufer enthalten Kohlensäure;“ "die Gasfüllung beginnt nach vollkommener Consolidirung des Knochens; ; "die zelligen Elemente, welche im fötalen Knochen in diesen Hohlräumen nachzuweisen, sind scheinen später ganz oder bis auf geringe Reste $\mathrm{zu}$ schwinden;“

nur gegen diese Sätze will ich mit meinen vermittelst der Goldmethode gewonnenen Resultaten za Felde ziehen und meine Erfahrungen, welche ich in Bezug auf die Kartheit und Vergänglichkeit der Zellen gemacht habe, die z. B. ganz bedeutend unter der Einwirkung von Wasser leiden können, zu Hülfe nehmen.

Welche Wichtigkeit der unbedingte Nachweis von Zellen fur mich hat, wird sich im Verlauf dieser Arbeit ergeben.

Was die Natur der Knochenhöhle anbetrifft, so vermag die Anwendung des Goldes kein neues Faktum beizubringen nach den erschöpfenden Untersuchungen L. Neu mann's über diesen Gegenstand. Vielleicht, dass der nach Retraction der vergoldeten Zelle zwischen ihr und der Höhlenwand mit Luftbläschen gefüllte Raum ein neues bestätigendes Moment für den Unterschied zwischen Zelle und Knochenhohlraum darbietet.

Vielfach ist über die Gestalt der Zellen gestritten worden und Neumann macht mit Recht auf die Vergeblichkeit des Streites aufmerksam, da man sich zur Darstellung der Zellen nur der Methoden bedient hat, mittelst welcher man die Knochenhöhle auch isolirt. Ich habe einem feinen vergoldeten Knochenschnitt einen Tropfen Salzsäure oder Salpetersäure zugesetzt und nach längerem Einwirken der Säure das Schnittchen zerzupft; ob aber die isolirten sternförmigen Gebilde nun wirklich die Zellen waren, oder nur die eng urn die Zelle schliessende, nicht zerstörte Knochenwand, lasse ich dahingestellt. Beim Triton und Meerschweinchen meine ich aber doch für das Vorhandensein von sternförmigen Zellausläufern plaidiren zu müssen. Man sieht nümlich an feinen Schnitten das tief violette Zellprotoplasma in die Anfänge der Knochenkanälchen sich ohne Cnterbrechung fortsetzen. Ferner erhält man, wenn man der Rerluctionsflüssigkeit etwas mehr Essigsäure zusetzt und das Präparat mit stärkerer Vergrösserung betrachtet, bisweilen folgendes Bild: man sieht das Lumen eines Knochenkanälchens da, wo es mit 
breiterer Basis der Knochenhöhle entspringt, durch zwei schwarze Linien scharf begrenzt und. sich verschmälernd weiter ziehen; der schwarzen Linie folgt nach innen $\mathrm{zu}$ ein heller, bisweilen glänzender Raum und diesem schliessen sich die scharfen Conturen des violetten Zellfortsatzes an. Wie weit der Fortsatz reicht und ob er sich mit einem entgegenkommenden verbindet, darüber wage ich kein Urtheil zu fällen, wenngleich ich ihn in manchen Präparaten mit einer Spitze enden zu sehen glaubte.

Isolirpräparate in der oben angegebenen Weise angefertigt, befreien zuweilen Zellen thatsächlich aus ihrem Kerker, allein dies geschieht dann auf Kosten der Integrität der Zelle; man sieht dann die Kerne von einer gewissen Menge Protoplasma gänzlich oder nur zum Theil umgeben. Dieses Protoplasma aber macht dann an seiner äussern Grenze stets den Eindruck des Zerfetzten.

\section{Die Nerven.}

So viel bisher bekannt ist, besitzen alle zum Knochen gehörigen Gewebe, das Periost, die compacte Substanz, das Mark, ihre Nerven. Während jedoch für das Periost schon eine Endigungsweise festgestellt ist, nämlich die in Vater'sche Körperchen ${ }^{1}$ ), so weiss man über das Verhalten der Nerven in der compacten Substanz nicht viel mehr, als dass sie mit kleinen Arterien in dieselbe eindringen.

Ich versuchte nun den weitern Verlauf der Nerven genau nach derselben Methode zu prüfen, nach der ich die Zellen untersucht habe, nur dass ich mich dabei auf die Schädelknochen von Tritonen und die Schenkelknochen von Meerschweinchen beschränkte. Eine gute Vergoldung von Schenkelknochen des Frosches ist mir so selten gelungen - ich weiss nicht aus welchem Grunde - dass ich von einer Untersuchung der Nerven bei diesem Thier Abstand nahm.

Oft sieht man ein derberes Nervenstämmchen von $0,012 \mathrm{Mm}$. Dicke mit einer kleinen Arterie in die compacte Substanz eindringen und auf dem Gefäss durch den Havers'schen Kanal Iaufen.

1) Rauber, Vater'sche Körperch. der Bänder und Periostnerven. München 1865. - Ueber d. Nerv. d. Knochenhaut u. Knochen d. Vorderarms und Untersch. München 1868. 
Da wo dieser und das Gefäss sich theilen, bekommt das Stämmchen eine Anschwellung, aus welcher sich zwei feinere Fasern entwickeln, welche in leicht gebogenem Verlauf, je mit einem Gefäss, in verschiedenen Kanälen weiter ziehen.

In Kanälen von $0,03 \mathrm{M}$. Weite findet man noch Nervenfasern, die eine Dicke von 0,003-0,005 Mm. aufzuweisen haben. Sich immer mehr verschmälernd und zu immer feineren Hälften zerspalten, gelangt die Nervenfaser schliesslich in die feinsten Haversschen Kanäle, von etwa $0,018 \mathrm{Mm}$. Durchmesser und darunter.

Hier ist sie demn zu einem äusserst feinen varicösen Färlchen geworden, das noch an Stärke abnimmt, indem die tiefdunkel violetten Varikositäten immer mehr an Umfang verlieren und der Verbindungsfaden immer zarter wird.

Bisweilen findet man zwei Fasern mittlerer Grösse in einem Havers'schen Kanal.

Auffallend ist die spärliche Zahl varicöser Fasern zur Menge und Grösse der Stämmchen ersten Ranges, welche in die compacte Substanz dringen. Es ist wohl dem Umstande zu zuschreiben, dass die Vergoldung eine recht schwierige ist, hauptsächlich aber, dass in der That nur wenige Zweige das Endziel in den feinsten Havers'schen Kanälen erreichen und die meisten sich der Markhöhle zuwenden.

Bekannt nun mit den Untersuchungen von $\mathrm{K}$ ühne ${ }^{1}$ ), der die Nerven in dem Zellprotoplasma der Hornhautkörperchen aufgehen lässt, von $\mathrm{Eberth}^{2}$ ), der einen Zusammenhang von Nervenfasern mit den Bindegewebskörpern im Schwanze der Froschlarve behauptet, und besonders von Lipman $n^{3}$ ), der die Endigung der Nerven in den Nucleolis der sternförmigen Zellen der Cornea beschreibt, machte ich den Versuch, einen Zusammenhang zwischen den feinsten varicösen Fasern der Havers'schen Kanäle und den Zellen, welche in den Knochenhöhlen liegen, aufzufinden.

1) Kühne Untersuch. über Protoplasma a. Contractilität. Leipzig 1864.

2) Eberth Arch. für mikrosk. Anat. Bd. 3. Entwickl. d. Geweb. im Schwanze d. Froschlarve.

3) Lipmann Virch. Arch. Bd. 48. Ueber Endigung d. Nerv. im eigentl. Geweb. u. hint. Epith. d. Hornh. d. Frosches. - Auch vorher schon hatte Verfasser mir mündlich Mittheilungen gemacht. 
Es kann sich Niemand der Schwierigkeiten, welchen eine derartige Untersuchung in der compacten Substanz unterliegt, bewusster sein, wie ich, der ich schon hinlänglich mit der Herstellung der bisherigen Präparate zu thun hatte. Es kann auch Niemand weniger enthusiastisch die Resultate meiner Untersuchung begriissen; dennoch glaube ich das Gewonnene mittheilen zu müssen, um Mitarbeiter zu finden, welche für den Zusammenhang der Zellen mit den Nerven noch unumstösslichere Beweise erlangen, als mir zu erreicheu müglich war.

Ist man zur feinen varicösen Faser des feinsten Havers'schen Kanales gelangt, so muss sich die Untersuchung natürlich auf die Intercellularsubstanz des Knochens selbst beziehen. Selten leider findet man dieselbe ungefärbt, meist trifft man sie in allen Farbennüancen von hellroth $\mathrm{zu}$ blauroth $\mathrm{zu}$ blauviolett; mehr oder weniger feine Linien durchsetzen sie und durchziehen sie überall; ebenso beinerkt man kleinere und grössere, glänzende und tief dunkle Punkte, die zum Theil Niederschlägen irgend welcher Art, zum Theil durchschnitten von Knochenkanälchen, angehören. Als feste Gestalten in diesem Chaos, das noch der lamellöse Ball vermehren hilft, sieht man die Knochenhöhlen mit ihren unmittelbaren Ausläufern und Längs- und Querschnitte Havers'scher Kanäle.

Bei stärkster Vergrösserung gewinnt das Bild an Klarheit, indem man bequemer die mannigfach gekrümmten Kanälchen bis in ihre feinsten Ausläufer verfolgen kann, die oft nur noch alis dünne ausgebuchtete Fädchen erscheinen; indem man viele der erwähnten Punkte sicherer als Querschnitte der feinen KanäIchen erkennt.

Man sieht ausserdem noch hier und da sehr kleine, bisweilen röthlich glänzende Punkte in zienlich regelmässigen Abständen von einander auftauchen und bei scharfer Einstellung gewahrt man, dass diese Punkte durch eine dunkle aber äusserst zarte Linie verbunden sind. Die Richtung dieser Linie zeichnet sich durch ihre Starrheit und Geradheit vor der abspringenden gröberen Linie aus, welche die feinsten Knochenkanälchen bilden.

Dieses so beschaffene Fädchen kann man nun nach zwei Richtungen hin verfolgen.

Einmal trifft man ein derartiges Fädchen bisweilen in der Nähe einer Zelle. Man sieht die zwei, drei, höchstens vier glänzenden, durch 
die schwarze Linie verbundenen Punkte gerade anf die Zelle zulaufen. Ganz in ihrer Nähe aber erleidet das Verbindungsfädchen eine Theilung, welche ich stets aus dem letzten Punkt hervorgehen gesehen habe. Das eine, der nun noch eben bei haarscharfer Einstellung wahrnehmbaren Theilungsfïdchen, verschwindet bald; ich sah es nur selten die Grenze der Zellhöhle erreichen; das andre aber, dis mit ihm einen spitzen Winkel bildet, lässt sich unter günstigen Umständen bis zu einem der glänzenden Kernkürperchen verfolgen. Hauchförmig hebt es sich noch eben von seiner Umgebung $a b$.

Viel schwerer lässt sich die oben erwähnte Faser nach der andern Seite hin verfolgen.

Ich gestehe offen, lass ich trotz vieler Präparate, die ich angefertigt, nur zweimal in der Lage war, eine Verbindung dieser Faser mit einem feinen varicösen Endfäserchen des Havers'schen Kanals anzunehmen.

Ich beschreibe das eine dieser Präparate (Fig. 2). Der Schnitt hat ein kleines Havers'sches Kanülchen nahezu quer getroffen. In dem Lumen des Kanals tindet sich ausser geringer violetter Masse (Blutkörperchen etc.) eine feine varicöse Faser, der ich nach meinen Erfahrungen die Bedeutnug einer Nervenfaser zusprechen muss. Da, wo die Faser der Wandung des Kanals mit einer feinen Spitze scheinbar anliegt, gehen zwei der zarten eben beschriebenen Fädchen ab. Das eine verliert sich in der das Havers'sche Kanälchen unmittelbar umgebenden concentrischen Substanz, das andre lässt sich darüber hinaus noch verfolgen.

Ein zweites Präparat (Fig. 3) zeigt das von dem Endpunkt (ler varicösen Faser in das Zwischengewebe tretende Fädchen, eine kleine Strecke dem Kanal parallel ziehend.

In beiden Fällen ist der Einwand gegen die nervöse Natur der Fädchen kein geringer, wenn man behauptet. es können gerade an der Stelle, wo die varicöse Faser endet, feinste Knochenkanälchen münden, welche man dann die Substanz durchziehen sieht. Diesen Einwand ganz zu entkräften, bin ich nicht im Stande; dennoch will ich ganz objectiver Weise die Punkte anfuihren, durch welche sich mir diese Fädchen von Knochenkanälen zu unterscheiden scheinen.

Einmal ist es die ausserordentliche Feinheit der Fäden, welche man sonst nicht an Knochenkanälchen beobachtet hat. 
Dann ist es der gerade starre Verlauf bei gleicher Stärke, während Knochenkanälchen sich sowohl mannigfach zu winden und zu verästeln, wie stärker oder schwächer im Verlauf zu werden pflegen.

Ferner pflegen Knochenkanälchen nicht nach einer Richtung und in so regelmässigen Abständen Zweige zu entsenden, wie es der Fall sein müsste, wenn die hellen Pünktchen der Ausdruck von Querschnitten noch feinerer Aestchen wären. Eine andere Verästelung, wie diese etwaige ist aber nicht zu bemerken, mit Ausnahme der Endfäden. Ebenso wenig gelingt es, die starren Fädchen in ein zweifelloses Knochenkanälchen übergehen zu sehen.

Während die Knochenkanälchen mit einem weitern Lumen stets von der Zellhöhle aus beginnen und erst durch Verästlung zu ihrer Feinheit allmählig gelangen, findet bei den Fäden gerade das Umgekehrte statt, indem sie sich ganz in der Nähe der Zellen durch Theilung noch verfeinern.

Lässt man diese Differenzen geiten, wie sie sich namentlich so scharf in den Kopfknochen von Tritonen ausprägen, und nimmt man nicht gar zu Sprüngen und Rissen seine Zuflucht, die dann mit ausgezeichneter Regelmässigkeit in Gestalt und Charakter auftreten würden, so bleibt meines Erachtens nichts übrig, als diese Fäılchen und Endfädchen als nervöse Gebilde zu betrachten.

Berlin, den 10. October 1869.

\section{Erkläruug der Abbildungen.}

Fig. 1-2 zeigt Nerven, die mit den Havers'schen Kanälen ziehen. Fig. 2 u. 4 vom Meerschweinchen, Fig. 1 u. 3 von Triton.

Fig. 2 u. 3 bei a Fasern, die in die Zwischensubstanz treten und mit der varicösen Faser im Havers'schen Kanal in Verbindung stehen.

Fig. 5. 6. 7. 8 Knochenzellen in den Höhlen von Triton; zarte mit hellen Prukten besetzte Füden treten heran und theilen sich, der Endfaden läuft zum Kernkörperchen.

Fig. 9 u. 10 Knochenzelle nebst Höhle vom Meerschweinchen, in Fig. 10 Luftbläschen nehen der retrahirten Zelle. Feines Fädchen zum Kernkörperchen.

Fig. 11 vergoldete Knochenzellen vom Ochsen. 
rigt

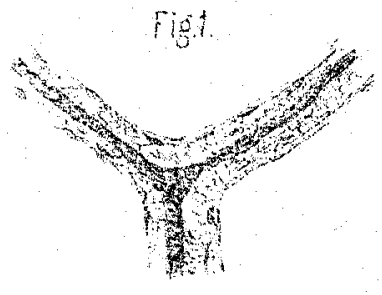

fig.

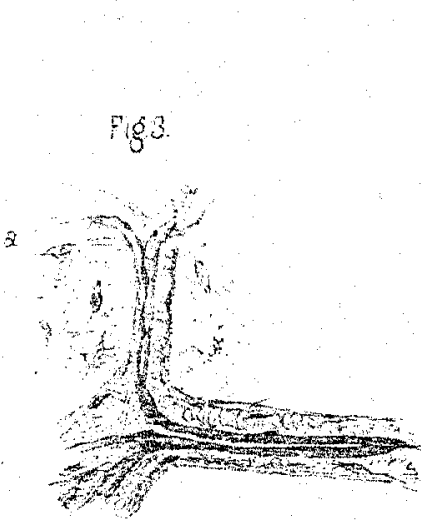

$\mathrm{Fig}^{4}$
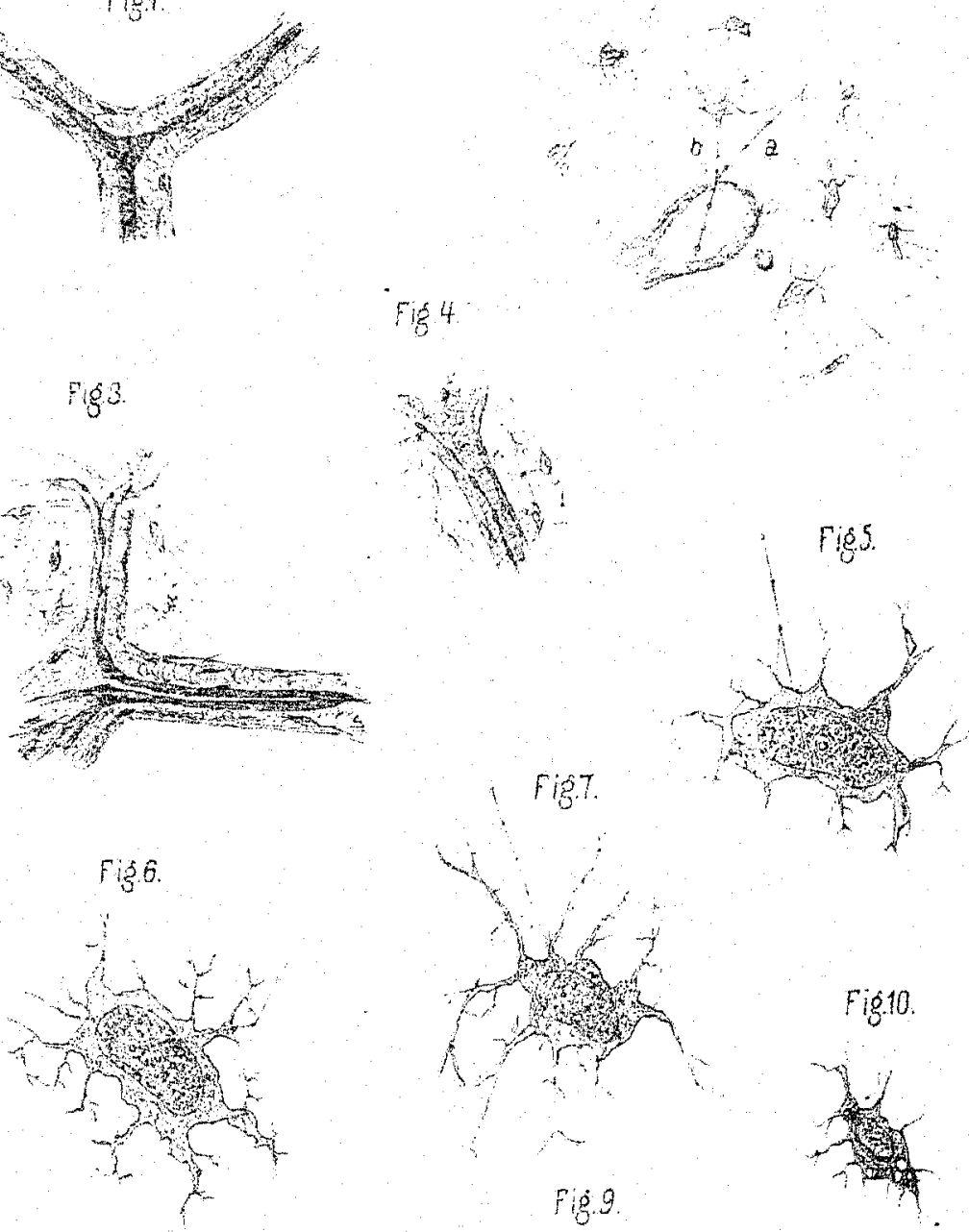

Fig.

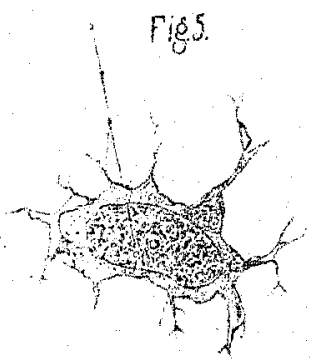

Fi $\$ .8$
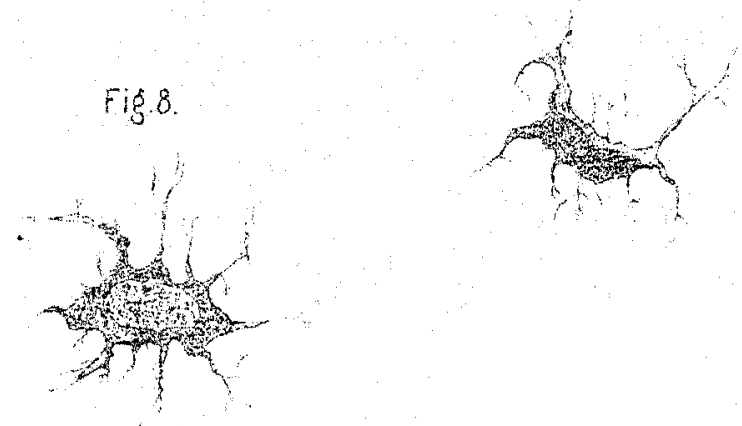

Fig10.

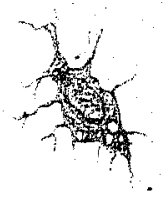

Fig.11.

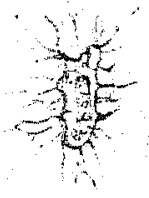

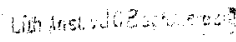

